\title{
EDITORIALS
}

\section{Exciting times ahead: a partnership with Nature Publishing Group}

\author{
Paul Stephenson, Aziz Sheikh \\ Editors-in-Chief, Primary Care Respiratory Journal \\ *Correspondence: c/o PCRJ Editorial Office, Unit 2, Warwick \\ House, Fairview Industrial Estate, Kingsbury Road, Curdworth, \\ Sutton Coldfield, B76 9EE, UK \\ Tel: +44 (0)1675 475401 Fax: +44 (0)1213361914 \\ E-mail: tricia@pcrs-uk.org
}

We are delighted to announce that the Primary Care Respiratory Journal $(P C R J)$ will be published by Nature Publishing Group (NPG) from 1st April this year. NPG have entered into a partnership agreement with the Primary Care Respiratory Society UK (PCRS-UK), the owners of the Journal, to re-launch the PCRJ under a new title brand. The rebranded $P C R J$ will continue as the official journal of the PCRS-UK and the International Primary Care Respiratory Group (IPCRG). PCRS-UK has worked in close collaboration with the IPCRG, as well as the editorial team, whilst negotiating the partnership agreement.

This partnership with NPG means that the journal will now benefit hugely from the strengths and capabilities of a top international academic publisher. There will be a change in title, to include the words 'respiratory medicine'. This is aimed at broadening the relevance of the journal to practitioners working in a range of international models of primary care, making it clear that we are interested in receiving contributions from clinicians and academics working within any sector that impacts on primary care management of respiratory and respiratory-related allergic diseases. The publishing partnership with NPG will be officially launched at the IPCRG conference in Athens, 21-24 May 2014.

This issue of the $P C R J$ will therefore be the final issue of the journal in its current form, and will be the last to have a hard copy issue. The rebranded journal will be online-only and fully openaccess, with an article processing charge (APC). This new business model for the journal will ensure sustainable access to all of our published content free of charge to readers anywhere in the world. This will help ensure longevity and sustainability in an environment of constant change, where funding agencies are more and more mandating publication of research in open-access journals.

The aim, of course, is to continue the progress made by the
$P C R J$ in recent years and to develop the journal into a world leader in its field. We obtained our first Impact factor of 2.191 last year, which ranked the $P C R J$ as $2 / 17$ among primary care journals and 30/50 among respiratory journals. Our internal calculations predict that our 2013 Impact factor, to be published in June 2014, will be above 2.5. NPG has the experience, expertise, worldwide reputation, global reach and infrastructure that the journal needs to develop further. The tendering, negotiating and planning process we have been through with NPG gives us every confidence that this is going to be a very successful partnership which will help to improve the quality of the end-product and increase its global reach. As such, we are very excited at the prospect of working with such an illustrious publisher.

There will be a seamless transfer from the current $P C R J$ publishing arrangements to the new publishing arrangements with NPG. Any articles submitted to the PCRJ up to the end of March 2014 will be automatically transferred over to the new title. Articles submitted to the journal from 1st April 2014 will be subject to the APC. We fully understand that the introduction of an APC, payable on article acceptance, is a new venture for this journal. Of course, authors from HINARI-band countries will have their APC waived, and other authors who cannot afford to pay and/or do not have a source of funding, can apply for a waiver if absolutely necessary. However, we hope that the Nature brand, the $P C R J$ 's reputation and well-established editorial processes, and our fast turnaround for submitted papers, should be very attractive to authors who wish to submit their work. In 2013, the average time to first decision for submitted papers was 23.7 days, and the time to final decision was 30.7 days.

Making the move to a new publisher is a major decision for any journal owner. Having worked very closely with the PCRS-UK we are well aware that considerable thought, expertise and negotiation has gone into this decision, which has been motivated by one core aim - ensuring that the journal continues to shape efforts to develop primary care respiratory medicine globally. It has been our pleasure to have been part of these deliberations and to work alongside a number of talented and highly committed individuals who have brought this agreement to fruition. They include: Anne Smith and Patrick White (representing the PCRS-UK); Siân Williams (representing the IPCRG); Mark Ware (an independent editorial consultant); Pooja Aggarwal and Joe Bennett (of NPG), and Richard Smith, Dermot 
Ryan, John Haughney and Wisia Wedzicha (who provided very helpful strategic advice).

As ever, we are extremely grateful to our superb team of assistant, statistical, section and associate editors, as well as the PCRS-UK and IPCRG representatives and everyone on the PCRJ International Editorial Board, for their support and guidance. We must also thank everyone who has contributed their time and expertise to review manuscripts for the PCRJ in 2013; this work, undertaken despite very busy schedules by experts in their field, is fundamental to our success in publishing high quality peerreviewed articles, and we are enormously grateful. The list of PCRJ 2013 reviewers is available online at www.thepcrj.org as an appendix to this editorial.
Finally, we would like to record our debt of gratitude to the team in the $P C R J$ editorial office, who have provided us with such stalwart support over many years. Led by Tricia Bryant and ably supported by Gail Ryan, Helen McDonnell, Liz Stockman, and Lynn Danzig, they have been the backbone of the journal. The success of the PCRJ and its continued development is in no small part a tribute to their dedication and hard work.

Conflicts of interest The authors declare that they have no conflicts of interest in relation to this article.

Online 28th February 2014

(C) 2014 Primary Care Respiratory Society UK. All rights reserved http://dx.doi.org/10.4104/pcrj.2014.00022 Prim Care Respir J 2014; 23(1): 1-2

\section{Vitamin D and the HLA locus help to explain the relationship between autoimmune and allergic diseases}

\section{See linked article by Maas et al. on pg 14}

\section{*Scott T Weiss ${ }^{1}$}

1 Professor of Medicine, Harvard Medical School; Associate Director, Channing Division of Network Medicine, Brigham and Women's Hospital, Boston MA, USA

*Correspondence: Professor Scott T Weiss, Professor of Medicine, Harvard Medical School, Department of Medicine, Brigham and Women's Hospital, Boston MA 02115, USA Tel: +617525 2278 Fax: +617525 4488

E-mail: scott.weiss@channing.harvard.edu

Autoimmunity has been defined as a T-helper cell type $1(\mathrm{Th} / 1)$ immune phenomenon, which creates an inflammatory response in the body against normal body substances (tissues/proteins). Allergy on the other hand is a T-helper cell type $2(\mathrm{Th} / 2)$ immune response to foreign antigen that also leads to inflammation. Because these two classes of immune disorders involve different clusters of differentiation 4 (CD4+) T-helper cell subsets, hitherto they have been considered immunologically separate and distinct - i.e. not caused by the same underlying genetic and environmental factors. However, in this issue of the PCRJ, Maas and colleagues have studied the co-occurrence of these immune diseases in families in the general population of the Netherlands, and have found that a diagnosed autoimmune disease in the parents suggested a roughly 2-fold increased risk of allergic disease in the eldest child. ${ }^{1}$ Their data suggest that there may be more of a common aetiology for these two types of immune disorders than previously thought. 2,3

Critical to the interpretation of the paper by Maas et al. is the issue of disease classification. The investigators used the International Classification of Primary Care (ICPC) diagnoses to define autoimmune disease in the parents. This system identifies the seven most common autoimmune diseases: psoriasis, rheumatoid arthritis/ankylosing spondylitis, ulcerative colitis/Crohn's disease, diabetes mellitus type 1, and multiple sclerosis. There are many more autoimmune diseases that were excluded from consideration because they are rare and hence not included in the ICPC. Including these rarer disorders might have made the results of the study even stronger. The allergic diseases classified included allergic asthma, hay fever, atopic dermatitis, and atopic conjunctivitis. This disease classification was relatively complete and thus misclassification bias is unlikely to have occurred for this outcome. Another methodological concern is that non-paternity was assessed and judged to be at an acceptable level; again, more stringent criteria for non-paternity would only have strengthened the results.

As noted by the authors, ${ }^{1}$ epidemiological data suggesting familial aggregation of these types of immune disorders in families is conflicting, and for some studies, statistical power has been limited by the low prevalence of the autoimmune diseases. Again, as noted by the authors, small sample size was an issue in some of the prior studies, as well as incomplete ascertainment of autoimmune disease. ${ }^{4.5}$ In contrast to the autoimmune diseases, the prevalence of allergy is high, and thus familial aggregation can occur simply by high disease prevalence alone.

The central question is whether this disease co-occurrence is just due to the nonspecific "environmental and epigenetic and genetic effects" alluded to by Maas and colleagues, ${ }^{1}$ or is there a hypothesis that might explain the underlying co-occurrence of autoimmune and allergic diseases in families?

I think there is a hypothesis as to what has caused a rise in prevalence of both Th1- and Th2-mediated immune diseases. First, the rise in both Th2 and Th1 immune diseases suggests that the immunological defect is at the next level up from the CD4 +, Th1, and Th2 cell, namely at the level of the T-regulatory cell (T-reg); these cells control both Th2 and Th1 inflammatory responses. These T-reg cells are in turn under the control of vitamin D, corticosteroids, and the cellular cytokines Transforming Growth factor Beta one (TGFB1) and 